\title{
Déclaration concernant la méningococcie et les voyageurs internationaux
}

McCarthy $A^{1}$, au nom du Comité consultatif de la médecine tropicale et de la médecine des voyages (CCMTMV)*

'L'Hôpital d'Ottawa, Campus Général, Ottawa (Ontario)

*Correspondance : CATMAT.Secretariat@phac-aspc.gc.ca

\section{Résumé}

Contexte : La méningite à méningocoques est présente partout dans le monde et les sérogroupes prédominants varient en fonction des régions géographiques. Des vaccins contre le méningocoque des sérogroupes $A, B, C, Y$ et W-135 sont offerts au Canada.

Objectif : Fournir des lignes directrices à l'intention des professionnels de la santé en matière de prévention de la méningococcie invasive chez les voyageurs internationaux du Canada.

Méthodologie : Cette déclaration a été préparée par le Comité consultatif de la médecine tropicale et de la médecine des voyages (CCMTMV) et se veut un complément au Guide canadien d'immunisation. Elle prend en considération la nécessité d'une protection, de même que les effets indésirables possibles de la vaccination.

Résultats : Les recommandations relatives au vaccin contre le méningocoque varient en fonction des caractéristiques du voyageur et de sa destination. La méningite à méningocoques est présente partout dans le monde et le sérogroupe prédominant varie en fonction des régions géographiques. Les régions qui présentent un risque particulier sont la " ceinture de méningite » de l'Afrique subsaharienne, l'Arabie saoudite pendant les pèlerinages Hadj et Oumra et les endroits où sévissent des épidémies ou qui enregistrent une forte incidence de la maladie. Pour que les voyageurs demeurent en bonne santé, veuillez consulter le Guide canadien d'immunisation. Un vaccin quadrivalent doit être administré aux personnes présentant un risque accru de méningococcie invasive en raison de problèmes médicaux, accompagné de doses de rappel tous les cinq ans. On devrait envisager d'administrer le vaccin contre le méningocoque B.

Conclusion : La vaccination est le moyen le plus efficace de prévenir la méningococcie invasive. Les Conseils de santé aux voyageurs du gouvernement du Canada servent à définir les zones d'activité nouvelle et récente du méningocoque et sont mis à jour régulièrement.

\section{Préambule}

Le Comité consultatif de la médecine tropicale et de la médecine des voyages (CCMTMV) donne de façon continue à l'Agence de la santé publique du Canada des conseils opportuns de nature médicale, scientifique et sanitaire concernant les maladies tropicales infectieuses et les risques pour la santé associés aux voyages internationaux. L'Agence reconnaît que les recommandations et les conseils formulés dans cette déclaration reposent sur les pratiques médicales et les connaissances scientifiques les plus récentes et les diffuse dans le but d'informer les voyageurs ainsi que les professionnels de la santé qui sont appelés à leur prodiguer des soins.

Les personnes qui administrent ou utilisent des médicaments, des vaccins ou d'autres produits devraient bien connaître la monographie des produits, ainsi que toute autre norme ou instruction approuvée concernant leur usage. Les recommandations relatives à l'usage des produits et les autres renseignements présentés ici peuvent 
différer de ceux qui figurent dans la monographie ou toute autre norme ou instruction approuvée pertinente établie par les fabricants autorisés. Les fabricants font approuver leurs produits et démontrent l'innocuité et l'efficacité de ceux-ci uniquement lorsque ces produits sont utilisés conformément à la monographie ou à toute autre norme ou instruction approuvée semblable.

\section{Introduction}

La présente déclaration a pour objectif de fournir des lignes directrices à l'intention des professionnels de la santé en matière de prévention de la méningococcie chez les voyageurs internationaux du Canada.

\section{Méthodologie}

Cette déclaration a été élaborée par un groupe de travail du Comité consultatif de la médecine tropicale et de la médecine des voyages (CCMTMV). Elle a été élaborée comme complément à la révision approfondie de la documentation et à l'analyse effectuée qui ont servi à formuler les recommandations du Comité consultatif national de l'immunisation (CCNI) présentées dans le Guide canadien d'immunisation (1). Le CCMTMV a pris en considération la nécessité d'une protection, de même que les effets indésirables possibles de la vaccination. La déclaration consiste en un examen narratif de la littérature sur la médecine santé-voyage liée aux vaccins contre le méningocoque et de l'opinion des experts du CCMTMV. Les recommandations ne comprennent pas une description de la fermeté de la recommandation ou de la qualité des données probantes, comme cela a été fait dans les déclarations précédentes du CCMTMV. Chaque membre du CCMTMV est bénévole, et aucun d'entre eux n'a signalé de conflit d'intérêts pertinent.

\section{Contexte}

La méningococcie est causée par la bactérie gram négative Neisseria meningitidis. Les bactéries Neisseria sont classées en 12 sérogroupes en fonction de la réactivité immunologique de leur polysaccharide capsulaire (2). Les cinq principaux sérogroupes les plus couramment associés à la maladie invasive sont les sérogroupes $A, B, C, Y$ et $\mathrm{W}-135(2,3,4)$.

La transmission interhumaine se produit par un contact étroit avec les sécrétions respiratoires ou la salive de personnes infectées $(1,4)$. L'homme est le seul réservoir (5). Le portage asymptomatique survient et, à tout moment, de $5 \%$ à $10 \%$ de la population peut être porteuse de $N$. meningitidis $(1,3,4)$. La méningococcie invasive est une conséquence rare de la colonisation du nasopharynx (6).

La méningococcie invasive survient généralement de 1 à 14 jours après une exposition et se caractérise habituellement par une maladie fébrile aiguë d'apparition soudaine associée à des caractéristiques de méningite ou de septicémie (méningococcémie), ou les deux, et à une éruption pétéchiale ou purpurique (sans blancheur) caractéristique. Les symptômes d'une méningite à méningocoques sont notamment d'intenses céphalées, de la fièvre, des nausées, des vomissements, une photophobie et des raideurs au niveau de la nuque. La méningococcémie entraîne souvent une hypotension, une insuffisance rénale aiguë, une hémorragie et la défaillance de nombreux organes (1,4). Le taux de mortalité est environ 5 à $10 \%$, même avec un traitement antimicrobien immédiat dans un établissement de soins de santé (5). Jusqu'à un tiers des survivants conservent des séquelles durables, notamment une perte auditive, diverses atteintes neurologiques et l'amputation de doigts ou de membres $(1,2,4)$.

\section{Épidémiologie}

N. meningitidis est présente partout dans le monde. Dans la plupart des pays, $N$. meningitidis est reconnue comme étant l'une des principales causes de la méningite et de la septicémie fulminante, et elle constitue un problème de santé publique important. Cependant, la surveillance de la population, la confirmation en laboratoire et la caractérisation des souches ne sont toujours pas réalisables dans de nombreux pays du monde (7). Les 
données de surveillance de nombreux pays sont incomplètes ou manquantes et il n'existe actuellement aucune estimation fiable du fardeau mondial (8).

La méningite à méningocoques est présente partout dans le monde et le sérogroupe prédominant varie en fonction des régions géographiques. En Australie, en Nouvelle-Zélande et en Europe, le sérogroupe $B$ prédomine, suivi du sérogroupe $C(7,9)$. Les sérogroupes $B$ et $C$ prédominent aux États-Unis et au Canada, suivis du sérogroupe $Y(7,9)$. La distribution des sérogroupes en Amérique latine, en Amérique du Sud et dans les Caraïbes varie. Les sérogroupes $B$ et $C$ prédominent dans certains pays tandis que les sérogroupes $\mathrm{W}-135$ et Y prédominent dans d'autres (9). On connaît peu de choses sur l'épidémiologie de la méningococcie en Asie et dans les régions avoisinantes $(7,9)$.

Différents profils de méningococcie invasive sont observés dans l'ensemble de l'Afrique (7). C'est le cas dans la ceinture de méningite africaine, une région de l'Afrique subsaharienne qui s'étend d'ouest en est, du Sénégal à l'Éthiopie (Figure 1) et dont la population compte environ 400 millions d'habitants (10). Pendant la saison sèche (de décembre à juin approximativement), le taux d'incidence de méningococcie peut atteindre jusqu'à 1000 cas pour 100000 habitants. Durant les mois pendant lesquels l'épidémie ne sévit pas, le taux de méningococcie dans cette région est d'environ 5 à 10 cas pour 100000 habitants $(4,8)$. Le risque est le plus élevé chez les voyageurs qui se rendent dans la ceinture de méningite et qui ont des contacts prolongés avec la population locale lors d'une épidémie.

En raison des conditions de promiscuité au cours des pèlerinages Hadj et Oumra en Arabie saoudite et du taux élevé des porteurs de $N$. meningitidis parmi les pèlerins, les éclosions de méningococcie sont depuis longtemps problématiques $(4,11)$. Les pèlerinages Hadj en 2000 et en 2001, par exemple, ont été associés à des éclosions importantes du sérogroupe $\mathrm{W}-135$ chez les pèlerins de retour au pays et chez leurs contacts.

Bien que les personnes de tout âge puissent contracter la maladie, la maladie endémique survient le plus souvent chez les enfants et les adolescents. Lorsque sévissent des épidémies de méningococcie cependant, les taux peuvent augmenter chez les enfants plus âgés et les jeunes adultes $(1,8)$. 
Figure 1 : Carte de la ceinture de méningite africaine ${ }^{1}$

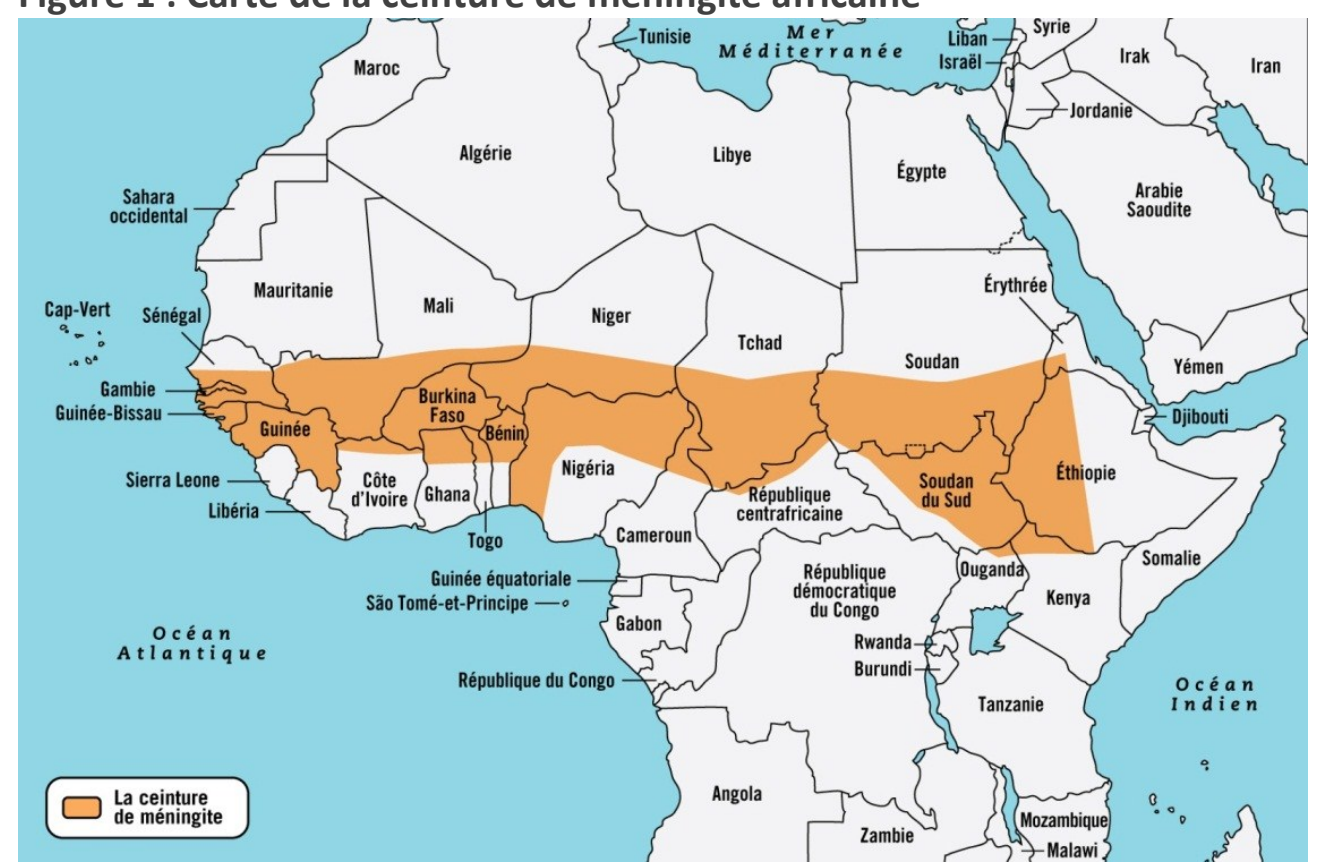

Source : Centers for Disease Control and Prevention (CDC). CDC Health Information for International Travel 2014 (The Yellow Book). Chapter 3: Meningococcal Disease.

http://wwwnc.cdc.gov/travel/yellowbook/2014/chapter-3-infectious-diseases-related-to-travel/meningococcal-disease (4)

\section{Prévention}

La vaccination est le moyen le plus efficace pour prévenir la méningococcie invasive. Des vaccins contre le méningocoque des sérogroupes $A, B, C, Y$ et $W-135$ sont offerts au Canada. Une description complète des vaccins contre le méningocoque des sérogroupes $A, C, Y$ et $W-135$ accompagnée de recommandations est présentée dans la version la plus récente du Guide canadien d'immunisation (1). Une description complète du vaccin contre le méningocoque du sérogroupe B est consignée dans la déclaration du CCNI sur les conseils relatifs à l'utilisation du vaccin 4CMenB (9).

\section{Recommandations en matière de vaccins pour les voyageurs canadiens}

Les recommandations relatives au vaccin contre le méningocoque varient en fonction des caractéristiques du voyageur (p. ex. âge, affections médicales, etc.) et de la destination. Les recommandations propres aux voyageurs canadiens sont décrites ci-dessous. Pour les voyageurs en bonne santé se rendant à une destination où le risque de transmission de la méningococcie est élevé, veuillez consulter le Tableau 1 dans la Partie 4 , Vaccins actifs, Vaccin contre le méningocoque pour obtenir le calendrier de vaccination contre le méningocoque du Guide canadien de l'immunisation (1).

Des programmes de vaccination systématique comprenant le vaccin conjugué contre le méningocoque du sérogroupe $\mathrm{C}$ ont été mis en place dans chaque province et territoire du Canada (1). Peu importe son programme de voyage, la vaccination du voyageur doit être à jour pour son âge, conformément au calendrier d'immunisation provincial ou territorial (12). 


\section{Voyageurs qui ont des problèmes médicaux sous-jacents}

La vaccination est recommandée pour les enfants et les adultes qui présentent un risque accru de méningococcie invasive, peu importe la destination (Tableau 1). Se reporter au Guide canadien d'immunisation pour obtenir des renseignements détaillés sur les produits recommandés, l'établissement du calendrier et la posologie (1).

\section{Tableau 1 : Recommandations relatives au vaccin contre le méningocoque pour les voyageurs qui} présentent un risque accru de contracter la méningococcie invasive en raison de problèmes médicaux $(1,2,9)$

\begin{tabular}{|c|c|c|}
\hline \multirow{2}{*}{$\begin{array}{c}\text { Sujets présentant un risque } \\
\text { accru de contracter la } \\
\text { méningococcie invasive en } \\
\text { raison de problèmes } \\
\text { médicaux }\end{array}$} & \multicolumn{2}{|c|}{ Recommandations relatives au vaccin } \\
\hline & Sérogroupes A, C, Y, W-135 & Sérogroupe B \\
\hline $\begin{array}{l}\text { Les personnes : } \\
\text { - } \quad \text { souffrant d'asplénie } \\
\text { fonctionnelle ou anatomique } \\
\text { (y compris une anémie } \\
\text { falciforme); } \\
\text { - } \quad \text { qui présentent un déficit } \\
\text { congénital en complément, } \\
\text { en properdine, en facteur D, } \\
\text { ou en anticorps primaires; } \\
\text { - qui présentent un déficit } \\
\text { acquis en complément } \\
\text { (p. ex. personnes recevant } \\
\text { de l'éculizumab). } \\
\text { En outre, l'immunisation devrait } \\
\text { être envisagée pour les } \\
\text { personnes atteintes d'une } \\
\text { infection au VIH, en particulier si } \\
\text { cette infection est congénitale, } \\
\text { et pour les personnes qui } \\
\text { portent un implant cochléaire. }\end{array}$ & $\begin{array}{l}\text { Sujets âgés de } 2 \text { mois à } \\
\text { moins de } 2 \text { ans } \\
{ }^{1} \\
\text { Men-C-ACYW-CRM } \\
\text { (Menveo }{ }^{\text {MC }} \text { ) doit être utilisé de } \\
\text { préférence. } \\
\text { Un calendrier de vaccination } \\
\text { comprenant } 2 \text { ou } 3 \text { doses est } \\
\text { recommandé pour les } \\
\text { nourrissons de } 2 \text { mois à moins } \\
\text { de } 1 \text { an, chaque dose étant } \\
\text { administrée à } 8 \text { semaines } \\
\text { d'intervalle, ainsi qu'une autre } \\
\text { dose lorsque le nourrisson est } \\
\text { âgé de } 12 \text { à } 23 \text { mois (et au } \\
\text { moins } 8 \text { semaines après } \\
\text { l'administration de la dose } \\
\text { précédente). } \\
\text { Pour les enfants de } 1 \text { an à } \\
\text { moins de } 2 \text { ans, } 2 \text { doses sont } \\
\text { recommandées, chaque dose } \\
\text { étant administrée à au moins } \\
8 \text { semaines d'intervalle. }\end{array}$ & $\begin{array}{l}\text { Sujets âgés de } 2 \text { mois à } \\
17 \text { ans } \\
\text { L'utilisation du vaccin } \\
\text { multicomposant contre le } \\
\text { méningocoque du } \\
\text { sérogroupe } B \text { (4CMenB) doit } \\
\text { être envisagée. } \\
\text { Il est recommandé } \\
\text { d'administrer } 3 \text { doses aux } \\
\text { nourrissons âgés de } 2 \text { à } \\
5 \text { mois, à au moins } 1 \text { mois } \\
\text { d'intervalle entre les doses. } \\
\text { Une quatrième dose (dose de } \\
\text { rappel) est recommandée } \\
\text { entre l'âge de } 12 \text { et de } \\
23 \text { mois. } \\
\text { Les } 2 \text { premières doses doivent } \\
\text { être administrées aux } \\
\text { nourrissons âgés de } 6 \text { à } \\
11 \text { mois à } 2 \text { mois d'intervalle et } \\
\text { une troisième dose est } \\
\text { recommandée entre l'âge de } \\
12 \text { et de } 23 \text { mois, au plus tôt } \\
2 \text { mois après la deuxième } \\
\text { dose. } \\
\text { Il est recommandé } \\
\text { d'administrer aux enfants âgés } \\
\text { de } 1 \text { à } 10 \text { ans, } 2 \text { doses à } \\
2 \text { mois d'intervalle. } \\
\text { II est recommandé } \\
\text { d'administrer aux enfants âgés } \\
\text { de } 11 \text { à } 17 \text { ans, } 2 \text { doses à au }\end{array}$ \\
\hline
\end{tabular}




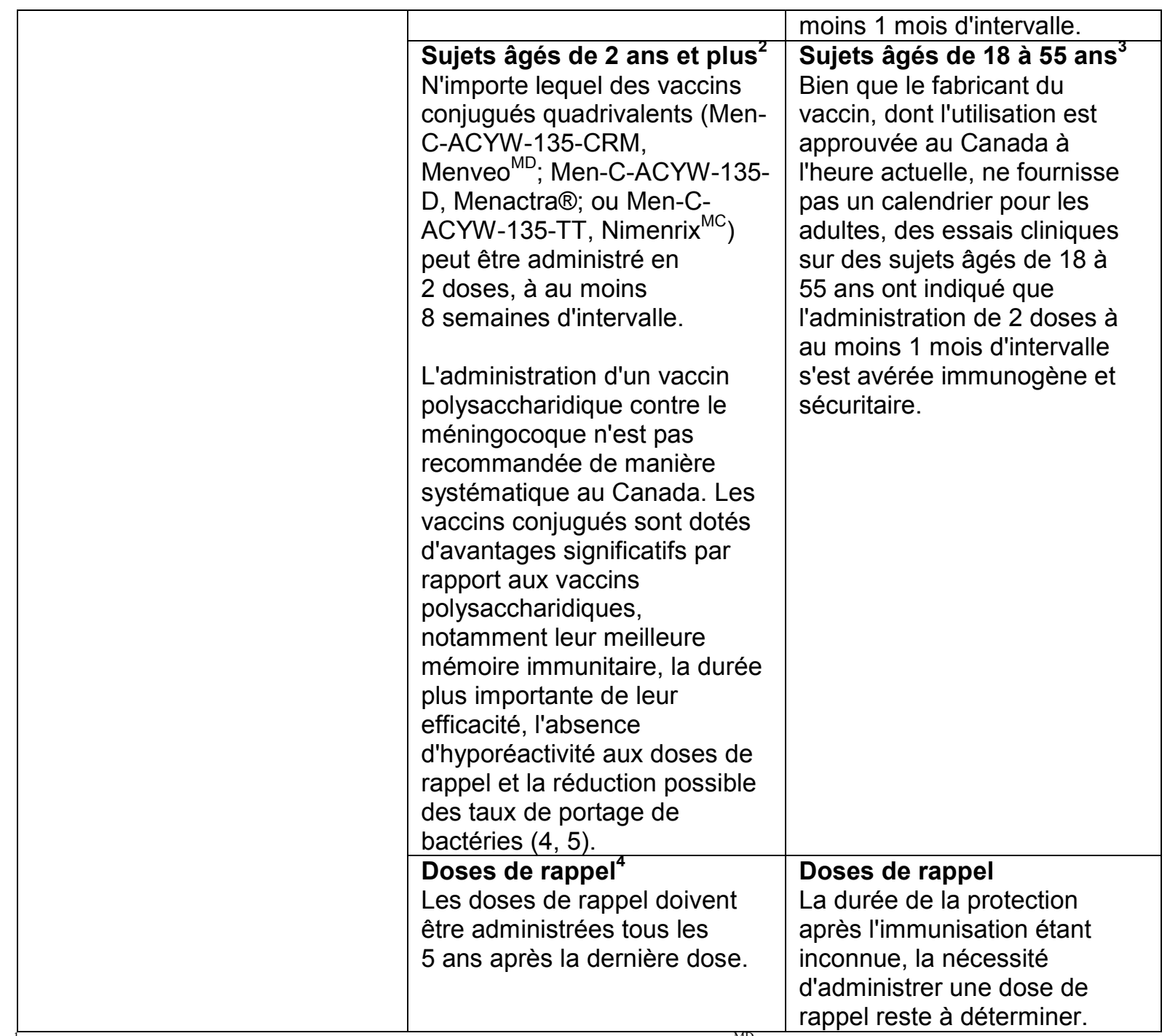

'Selon les données publiées offertes pour ce groupe d'âge, le vaccin à utiliser est Menveo ${ }^{\mathrm{MD}}$ en raison de son innocuité et de son immunogénicité éprouvées. Le vaccin conjugué contre le méningocoque du sérogroupe $\mathrm{C}$ n'a pas à être administré de façon systématique en plus du vaccin Menveo ${ }^{\mathrm{MD}}$.

${ }^{2}$ Les vaccins Men-C-ACYW-135 ne sont pas approuvés pour les personnes de 56 ans et plus; cependant, selon certaines données limitées et l'avis des experts, leur utilisation est considérée comme appropriée (2).

${ }^{3} \mathrm{Au}$ Canada, l'utilisation du vaccin $4 \mathrm{CMenB}$ a été autorisée pour les sujets âgés de 2 mois à 17 ans.

${ }^{4}$ Le fabricant de Nimenrix ${ }^{\mathrm{MC}}$ n'a pas encore déterminé la nécessité d'une dose de rappel; cependant, le CCNI recommande des doses de rappel périodiques pour les personnes qui présentent un risque élevé de méningococcie invasive ou qui courent un risque continu d'exposition (2).

\section{Voyageurs qui entreprennent les pèlerinages Hadj et Oumra}

À la suite de deux grandes éclosions de méningococcie invasive (MI) au cours des pèlerinages de 2000 et de 2001, le ministère de la Santé de l'Arabie saoudite exige désormais que tous les pèlerins reçoivent un vaccin contre le méningocoque. Tous les visiteurs qui se présentent dans le but d'effectuer le Hadj ou la Oumra doivent fournir un certificat de vaccination contre la méningite à méningocoques. Les visas pour le Hadj ou la Oumra sont refusés sans preuve de vaccination valide.

- Les adultes et les enfants âgés de deux ans et plus doivent recevoir le vaccin quadrivalent contre le méningocoque (sérogroupes A, C, Y et W-135) (13). 
- Les enfants âgés de trois mois à deux ans doivent recevoir deux doses du vaccin contre le méningocoque, à trois mois d'intervalle entre les deux doses (13).

- La vaccination doit avoir été reçue pas plus de trois ans et pas moins de dix jours avant leur arrivée en Arabie saoudite (13).

En général, les voyageurs qui se rendent dans cette région n'ont pas besoin de se faire administrer le vaccin $4 \mathrm{CMenB}$, sauf s'il existe des données probantes concernant la présence d'une souche hyperendémique ou d'une éclosion de méningocoque attribuable au sérogroupe $B$, qui peuvent être évitées grâce à ce vaccin.

Veuillez consulter les recommandations dans les Conseils de santé aux voyageurs du gouvernement du Canada propres aux pèlerins qui entreprendront le Hadj et la Oumra. Ils sont publiés chaque année à la fin de l'été et au début de l'automne et donnent des renseignements à jour pour s'assurer que chaque voyageur possède les documents de vaccination pertinents (14).

\section{Voyageurs se rendant en Afrique subsaharienne}

Le vaccin conjugué quadrivalent contre le méningocoque (sérogroupes $A, C, Y$ et $W-135$ ) est recommandé pour les voyageurs qui se rendent dans la ceinture de méningite africaine (Figure 1) ou dans les pays situés en dehors des limites habituelles et dans lesquels des épidémies ont été observées. Cette recommandation s'applique en particulier aux personnes qui vivront ou qui travailleront dans ces régions, ou qui seront en contact étroit avec la population locale (école, lieu de résidence, etc.)

L'immunisation contre le seul sérogroupe $C$ n'est pas considérée comme étant adéquate pour les personnes qui se rendent dans cette région. Une dose unique de n'importe lequel des vaccins conjugués quadrivalents contre le méningocoque peut être administrée pour l'immunisation des personnes âgées de deux ans et plus (2). Le vaccin Men-C-ACYW-135-CRM (Menveo ${ }^{\mathrm{MD}}$ ) est recommandé pour l'immunisation des personnes âgées de deux mois à moins de deux ans. Se reporter au Guide canadien d'immunisation pour obtenir des renseignements détaillés et les calendriers (1).

En général, les voyageurs qui se rendent dans cette région n'ont pas besoin de se faire administrer le vaccin $4 C M e n B$, sauf s'il existe des données probantes concernant la présence d'une souche hyperendémique ou d'une éclosion de méningocoque attribuable au sérogroupe $B$, qui peuvent être évitées grâce à ce vaccin.

\section{Autres considérations pour les voyageurs}

- Les voyageurs qui se rendent dans les régions où des épidémies sont en cours ou dans lesquelles on observe une forte incidence de la maladie doivent être vaccinés, quelle que soit la durée de l'exposition. Les Conseils de santé aux voyageurs du gouvernement du Canada concernant les zones d'activité nouvelle et récente du méningocoque sont publiés et mis à jour régulièrement sur le site voyage.gc.ca (14).

- La méningococcie invasive est apparue traditionnellement dans les écoles, les collèges et autres endroits où étaient regroupés de grands nombres d'adolescents et de jeunes adultes. Les personnes qui se rendent dans ces milieux doivent envisager de recevoir le vaccin contre le méningocoque des sérogroupes $B$ et $A, C, Y, W-135$ au moins deux semaines avant leur arrivée (5).

- Les personnes qui se rendent dans des lieux de recherche, des laboratoires industriels ou cliniques et qui risquent d'être systématiquement exposées à $N$. meningitidis doivent recevoir le vaccin Men-C-ACYW135 et le vaccin 4 CMenB. Le personnel de laboratoire qui risque une exposition continue doit être revacciné à cinq d'intervalle, systématiquement (1). Le personnel de laboratoire qui risque une exposition continue doit être revacciné systématiquement à cinq ans d'intervalle avec le vaccin Men-C-ACYW-135 (1). La durée de la protection après l'immunisation étant inconnue, la nécessité d'administrer une dose de rappel du vaccin 4 CMenB reste à déterminer (9). 
- On ne dispose pas de données suffisantes pour recommander l'immunisation systématique contre le méningocoque pour les personnes qui se déplacent à titre de fournisseurs de soins de santé, la transmission nosocomiale de la méningococcie invasive étant très rare (1).

- Les voyageurs qui se rendent dans des pays développés doivent suivre les recommandations en matière de vaccination contre le méningocoque du pays de destination (15).

\section{Autres caractéristiques et utilisations du vaccin}

Le Tableau 2 fournit des renseignements supplémentaires sur les vaccins, tels que leurs effets secondaires courants, les contre-indications, les précautions et leur utilisation chez les populations spéciales. Pour obtenir des renseignements détaillés et exhaustifs, veuillez consulter le Guide canadien d'immunisation (1).

Tableau 2 : Autres caractéristiques des vaccins contre le méningocoque et recommandations pour leur utilisation

\begin{tabular}{|c|c|c|}
\hline Caractéristique du vaccin & $\begin{array}{l}\text { Vaccin contre le méningocoque } \\
\text { des sérogroupes } A, C, Y \text { et } W-135\end{array}$ & $\begin{array}{l}\text { Vaccin contre le méningocoque } \\
\text { du sérogroupe B }\end{array}$ \\
\hline Effets indésirables & $\begin{array}{l}\text { Réactions bénignes au point } \\
\text { d'injection ( } p . \text { ex. rougeur, } \\
\text { sensibilité, enflure) et réactions } \\
\text { systémiques ( } p . \text { ex. mal de tête, } \\
\text { malaise) signalées. Les effets } \\
\text { indésirables graves sont rares. }\end{array}$ & $\begin{array}{l}\text { Chez les nourrissons et les enfants } \\
\text { de moins de } 12 \text { mois, les effets } \\
\text { indésirables les plus souvent } \\
\text { signalés comprenaient l'érythème, } \\
\text { l'induration, la fièvre et la } \\
\text { somnolence ou l'irritabilité. Un } \\
\text { examen des données est consigné } \\
\text { dans la déclaration du CCNI sur les } \\
\text { Conseils relatifs à l'utilisation du } \\
\text { vaccin } 4 \text { CMenB (9). }\end{array}$ \\
\hline $\begin{array}{l}\text { Contre-indications et } \\
\text { précautions }\end{array}$ & $\begin{array}{l}\text { L'immunisation est contre-indiquée } \\
\text { pour les personnes qui ont déjà eu } \\
\text { une réaction anaphylactique à la } \\
\text { suite d'une vaccination, ou pour } \\
\text { celles qui ont une réaction } \\
\text { anaphylactique reconnue à l'un des } \\
\text { composants du vaccin ou de son } \\
\text { contenant (1). } \\
\text { L'administration du vaccin contre le } \\
\text { méningocoque devrait être retardée } \\
\text { pour les personnes atteintes d'une } \\
\text { maladie aiguë modérée ou grave. } \\
\text { Les personnes atteintes de } \\
\text { maladies aiguës mineures (avec ou } \\
\text { sans fièvre) peuvent être vaccinées } \\
\text { (1). }\end{array}$ & $\begin{array}{l}\text { Le vaccin est contre-indiqué chez } \\
\text { les personnes souffrant d'une } \\
\text { allergie grave à toute composante } \\
\text { du vaccin ou ayant présenté une } \\
\text { réaction allergique grave à une dose } \\
\text { antérieure (2). }\end{array}$ \\
\hline Grossesse et allaitement & $\begin{array}{l}\text { Le vaccin conjugué contre le } \\
\text { méningocoque n'a fait l'objet } \\
\text { d'aucune étude chez les femmes } \\
\text { enceintes. Cependant, son usage } \\
\text { peut être envisagé si nécessaire (1, } \\
\text { 3). Les vaccins inactivés, tels les }\end{array}$ & $\begin{array}{l}\text { Aucune étude du vaccin } 4 C M e n B \\
\text { n'a été réalisée auprès de femmes } \\
\text { enceintes ou qui allaitent. }\end{array}$ \\
\hline
\end{tabular}




\begin{tabular}{|l|l|l|}
\hline & $\begin{array}{l}\text { vaccins contre le méningocoque, } \\
\text { peuvent être administrés aux } \\
\text { femmes qui allaitent (1). }\end{array}$ & \multicolumn{1}{|l|}{} \\
\hline $\begin{array}{l}\text { Voyageurs } \\
\text { immunodéprimés }\end{array}$ & $\begin{array}{l}\text { Le vaccin contre le méningocoque est recommandé pour certaines } \\
\text { personnes à risque élevé, tel qu'il est décrit ci-dessus. Lorsqu'on envisage } \\
\text { de vacciner une personne immunodéprimée, une consultation auprès du } \\
\text { médecin traitant peut être utile. Il est conseillé d'adresser les cas } \\
\text { complexes à un médecin qui possède une expertise en immunisation ou en } \\
\text { immunodéficience. }\end{array}$ \\
\hline $\begin{array}{l}\text { Administration } \\
\text { concomitante d'autres } \\
\text { vaccins }\end{array}$ & $\begin{array}{l}\text { Les vaccins conjugués } \\
\text { quadrivalents peuvent être } \\
\text { administrés simultanément avec } \\
\text { d'autres vaccins adaptés aux } \\
\text { adolescents et aux adultes, à des } \\
\text { sites d'injection différents en } \\
\text { utilisant des seringues et des } \\
\text { aiguilles distinctes (1). }\end{array}$ & $\begin{array}{l}\text { Un examen de l'utilisation du vaccin } \\
\text { 4CMenB en concomitance avec } \\
\text { d'autres vaccins est consigné dans } \\
\text { la déclaration du CCNI sur les } \\
\text { Conseils relatifs à l'utilisation du } \\
\text { vaccin 4CMenB (9). }\end{array}$ \\
\hline $\begin{array}{l}\text { Interchangeabilité des } \\
\text { vaccins }\end{array}$ & $\begin{array}{l}\text { Tout les vaccins conjugués } \\
\text { quadrivalents peuvent être utilisés } \\
\text { pour une revaccination, et ce, peu } \\
\text { importe le vaccin } \\
\text { antiméningococcique qui avait été } \\
\text { administré au départ (1). La série } \\
\text { vaccinale administrée aux } \\
\text { nourrissons devrait être complétée } \\
\text { au moyen du même vaccin, dans la } \\
\text { mesure du possible. }\end{array}$ & Sans objet. \\
\hline
\end{tabular}

\section{Précautions générales}

II faut conseiller aux voyageurs d'adopter de bonnes pratiques d'hygiène des mains et d'éviter les activités visant à promouvoir l'échange de sécrétions respiratoires, notamment le partage de boissons, de cigarettes, de rouge à lèvres, etc. Il faut éviter le surpeuplement dans des espaces restreints. À la suite d'un contact étroit avec une personne infectée par la méningococcie, on doit chercher à obtenir des conseils médicaux au sujet d'une chimioprophylaxie et d'une vaccination possible (5).

\section{Conclusion}

La vaccination contre le méningocoque est le moyen le plus efficace de prévenir la méningococcie invasive. Les Conseils de santé aux voyageurs du gouvernement du Canada servent à définir les zones d'activité nouvelle et récente du méningocoque et sont mis à jour régulièrement (14).

\section{Remerciements}

La présente déclaration a été préparée par le groupe de travail sur le méningocoque : McCarthy A (présidente), Bryson M, Bui Y, Cutler J et Geduld J.

Membres du CCMTMV : McCarthy A (présidente), Boggild A, Brophy J, Bui Y, Crockett M, Greenaway C, Libman M, Teitelbaum P et Vaughan S. 
Membres de liaison : Gershman M (Centers for Disease Control and Prevention des États-Unis) et Pernica J (Association pour la microbiologie médicale et l'infectiologie Canada).

Membres d'office : Marion D (Centre des services de santé des Forces canadiennes, ministère de la Défense nationale), McDonald $\mathrm{P}$ (Division des médicaments anti-infectieux, Santé Canada), Schofield S (Direction de la protection de la santé de la Force, ministère de la Défense nationale) et Tepper $\mathrm{M}$ (Direction de la protection de la santé de la Force, ministère de la Défense nationale).

\section{Conflit d'intérêts}

Aucun

\section{Financement}

Ce travail a été appuyé par l'Agence de la santé publique du Canada.

\section{References}

(1) National Advisory Committee on Immunization (NACI). Canadian immunization guide. Part 4 - Active immunizing agents - meningococcal vaccine. Cat:: HP40-3/2014E ed. Ottawa (ON): Public Health Agency of Canada; 2014. http://www.phac-aspc.gc.ca/publicat/cig-gci/p04-meni-eng.php.

(disponible en français: http://www.phac-aspc.gc.ca/publicat/cig-gci/p04-meni-fra.php).

(2) National Advisory Committee on Immunization (NACl). An Advisory Committee Statement (ACS): Update on quadrivalent meningococcal vaccines available in Canada (unpublished), September 2014.

Une déclaration d'un comité consultatif (DCC). Comité consultatif national de l'immunisation (CCNI): Mise à jour sur l'utilisation des vaccins quadrivalents contre le méningocoque au Canada (non publié), septembre 2014.

(3) Heymann DL, editor. Control of communicable diseases manual 19th ed. Washington DC: American Public Health Association; 2008.

(4) Centers for Disease Control and Prevention (CDC). CDC Health information for international travel 2014 (Yellow Book). Chapter 3: Meningococcal Disease. Atlanta: CDC; 2014.

http://wwwnc.cdc.gov/travel/yellowbook/2014/chapter-3-infectious-diseases-related-to-travel/meningococcal-disease.

(5) World Health Organization. International travel and health 2012. Chapter 6: Vaccine-preventable diseases and vaccines.

http://www.who.int/ith/ITH_chapter_6.pdf?ua=1.

(6) Advisory Committee on Immunization Practices. Prevention and control of meningococcal disease:

Recommendations of the Advisory Committee on Immunization Practices (ACIP). MMWR. 2013;62(2).

(7) Halperin SA, Bettinger JA, Greenwood B, Harrison LH, Jelfs J, et al. The changing and dynamic epidemiology of meningococcal disease. Vaccine. 2012;30(SUPPL. 2):B26-36.

(8) World Health Organization. Meningococcal vaccines: WHO position paper, November 2011. Weekly Epidemiological Record. 2011;86:521-540. http://www.who.int/wer/2011/wer8647.pdf?ua=1.

(9) National Advisory Committee on Immunization. An Advisory Committee Statement (ACS): Advice for the use of the multicomponent meningococcal serogroup B (4CMenB) vaccine, 2014. Ottawa (ON): Public Health Agency of Canada; 2014. http://publications.gc.ca/collections/collection_2014/aspc-phac/HP40-104-2014-eng.pdf. (disponible en français: http://publications.gc.ca/site/fra/9.699195/publication.html).

(10) World Health Organization. Background paper on meningococcal vaccines SAGE Working Group. Geneva: WHO; 2011.

http://www.who.int/immunization/sage/1_mening_background_document_v5_3_apr_2011.pdf.

(11) European Centre for Disease Prevention and Control. Laboratory-confirmed invasive meningococcal disease: Effect of the Hajj Vaccination Policy, Saudi Arabia, 1995 to 2011. Eurosurveillance. 2014;19 (37).

(12) Government of Canada [Internet]. Provincial and territorial immunization information. Ottawa (ON): Government of Canada; 2015.

http://healthycanadians.gc.ca/healthy-living-vie-saine/immunization-immunisation/children-enfants/schedulecalendrier-eng.php\#a3. 
(disponible en français: http://canadiensensante.gc.ca/healthy-living-vie-saine/immunization-immunisation/childrenenfants/schedule-calendrier-fra.php?_ga=1.71561811.1575686084.1412886377).

(13) Kingdom of Saudi Arabia. The Ministry of Hajj Portal. http://haj.gov.sa/en-us/Pages/default.aspx.

(14) Government of Canada [Internet]. Travel Health Notices. Ottawa (ON): Government of Canada; 2015. http://travel.gc.ca/travelling/health-safety/travel-health-notices.

(disponible en français: http://voyage.gc.ca/voyager/sante-securite/conseils-sante-voyageurs).

(15) Public Health Agency of Canada. Committee to Advise on Tropical Medicine and Travel (CATMAT) Statement on Meningococcal Vaccination for Travellers, 2009. CCDR. June 2009;35(ACS -4).

http://www.phac-aspc.gc.ca/publicat/ccdr-rmtc/09vol35/acs-dcc-4/index-eng.php

(disponible en français: http://www.phac-aspc.gc.ca/publicat/ccdr-rmtc/09vol35/acs-dcc-4/index-fra.php). 